\title{
Soroprevalência de tripanossomíase americana em adultos de uma área da Amazônia ocidental Brasileira
}

\author{
Seroprevalence of American trypanosomiasis in adults in an \\ area of the western Brazilian Amazon region
}

\author{
Thor Oliveira Dantas-Maia ${ }^{1,3}$, Cleudson Castro $^{1}$, Alejandro Luquetti Ostermayer ${ }^{2}$ \\ e Vanize Macêdo ${ }^{1 \dagger}$
}

\begin{abstract}
RESUMO
Foi realizado no período de janeiro a março de 2001 um inquérito soroepidemiológico para tripanossomíase americana (doença de Chagas) em populações humanas, urbana e rural, de áreas da bacia do alto Purus, Amazônia Ocidental Brasileira, através de testagem em série utilizando três técnicas sorológicas. A amostra foi constituída de 1.055 indivíduos, 844 da área urbana e 211 da área rural. Foi identificada a infecção autóctone em nove indivíduos, cinco da área urbana e quatro da área rural, com idades variando entre 16 e 72 anos. As prevalências estimadas de infecção chagásica foram: 0,6\% (IC95\% 0,2-1,4) e 1,9\% (IC95\% 0,6-4,5) para as áreas urbana e rural respectivamente. Observou-se aparente tendência à aglomeração: sete eram naturais do rio Purus, três residiam na mesma localidade, dois habitavam o mesmo domicílio.
\end{abstract}

Palavras-chaves: Doença de Chagas. Amazônia Ocidental Brasileira. Soroepidemiologia. Inquérito.

\begin{abstract}
From January to March 2001 a seroepidemiological survey for American trypanosomiasis (Chagas disease) was carried out among urban and rural human populations in areas of the Upper Purus basin, in the western Brazilian Amazon region, using serial testing with three different serological techniques. The sample was composed of 1,055 individuals: 844 from urban and 211 from rural areas. Autochthonous infection was identified in nine individuals aged 16 to 72 years: five from urban and four from rural areas. The estimated prevalences of Chagas infection for the urban and rural areas were 0.6\% (95\% CI: 0.2-1.4) and 1.9\% (95\% CI: 0.6-4.5) respectively. An apparent clustering trend was noted: seven were born along the Purus river: three lived at the same locality and two of them in the same dwelling.
\end{abstract}

Key-words: Chagas' disease. Western Brazilian Amazon. Seroepidemiology. Survey.

A infecção chagásica expandiu-se durante a primeira metade do século XX até constituir-se em grave problema de saúde pública em grande parte do continente americano ${ }^{25}$, atingindo 16 a 18 milhões de infectados e causando apenas nos sete países do Cone Sul, uma perda estimada de 750 mil anos de vida por ano ${ }^{11}$. 0 bem sucedido programa de controle da doença no Brasil alcançou enorme impacto sobre indicadores entomológicos, sorológicos e de morbimortalidade, determinando importantes mudanças no quadro epidemiológico da endemia, que traz, no entanto, novos e permanentes desafios ${ }^{1920}$.

Nesse contexto a ocorrência da infecção chagásica na Amazônia Brasileira vem sendo considerada por alguns como um potencial novo desafio frente a esta patologia ${ }^{4813}$, desde que seu ciclo enzoótico foi elucidado ${ }^{2910}$ e os primeiros casos humanos autóctones foram diagnosticados ${ }^{17}$. Em aparente expansão, evidenciada por seus números crescentes ${ }^{22}$, tem merecido particular interesse e atenção a discussão acerca do risco de sua endemização na regiã $0^{46}$.

A vigilância entomológica tem demonstrado na Amazônia uma ampla e complexa distribuição de cerca de duas dezenas de espécies nativas de triatomíneos ${ }^{12}$, principalmente do gênero Rhodnius $^{18}$, além de situações particulares de domiciliação incipiente de Panstrongylus geniculatus ${ }^{21}$, de Triatoma maculata $^{16}$, e de transmissão silvestre por Rhodnius brethesi

\footnotetext{
1. Núcleo de Medicina Tropical, Universidade de Brasília, Brasília, DF. 2. Instituto de Patologia Tropical e Saúde Pública, Universidade Federal de Goiás, Goiânia, G0. 3. Departamento de Ciências da Saúde, Universidade Federal do Acre, Rio Branco, AC.

$\dagger$ Este artigo é dedicado In memoriam à Profa. Vanize Macêdo, que faleceu enquanto o trabalho era concluído.

Endereço para correspondência: Dr. Thor Oliveira Dantas-Maia. Fundação Hospital Estadual do Acre (FUNDHACRE). Rodovia BR-364, Km 2, s/n, Distrito Industrial. 69914-220 Rio Branco, AC.

Tel: 5568 3223-4824; Fax: 5568 3226-4321

e-mail: thor.dantas.maia@brturbo.com.br

Recebido em: 10/07/2006

Aceito em: 20/06/2007
} 
em piaçabais5. No Acre, há registros de Rhodnius robustus e Rhodnius pictipes, ambos infectados, em palmeiras próximas aos domicílios de casos agudos ${ }^{124}$.

0 inquérito sorológico nacional conduzido de 1975 a 1980 mostrava coeficientes de prevalência inesperadamente altos para dois estados da região norte: Amazonas com 1,9\% e Acre com 2,4\%. Aárea rural do município de Sena Madureira, no Acre apresentou prevalência de 3,7\%, (dado não publicado) tendo gerado a necessidade de estudos posteriores para confirmação de tais resultados ${ }^{3}$.

0 objetivo principal desse estudo, portanto foi o de realizar inquérito soroepidemiológico estimando os coeficientes de prevalência da tripanossomíase americana (doença de Chagas) em populações humanas adultas, das áreas urbana e rural do município de Sena Madureira, Estado do Acre e localidades rurais adjacentes do sudoeste do Estado do Amazonas, bacia do alto Purus, Amazônia Ocidental Brasileira. Um objetivo secundário foi o de investigar a presença de triatomíneos no peridomicílio e em ecótopos silvestres próximos, na área rural da região estudada.

\section{MATERIAL E MÉTODOS}

o Estado do Acre situa-se a norte e a oeste no território nacional, na porção ocidental-sul da Amazônia Brasileira (Figura 1), entre as latitudes de $07^{\circ} 07^{\prime}$ e $11^{\circ} 08^{\prime} \mathrm{S}$, e as longitudes de 66 $30^{\circ}$ e $74^{\circ} \mathrm{WGr}$. Limita-se ao norte com o Estado do Amazonas, a leste com o Estado de Rondônia, a sul e oeste com a República do Peru e a sudeste com a República da Bolívia. Sua superfície territorial é de $153.149,9 \mathrm{~km}^{2}$, correspondente a 3,9\% da área amazônica brasileira e a $1,8 \%$ do território nacional ${ }^{15}$.

0 município de Sena Madureira representa 16,5\% da área total do Estado do Acre. Situa-se na microrregião do Purus e faz

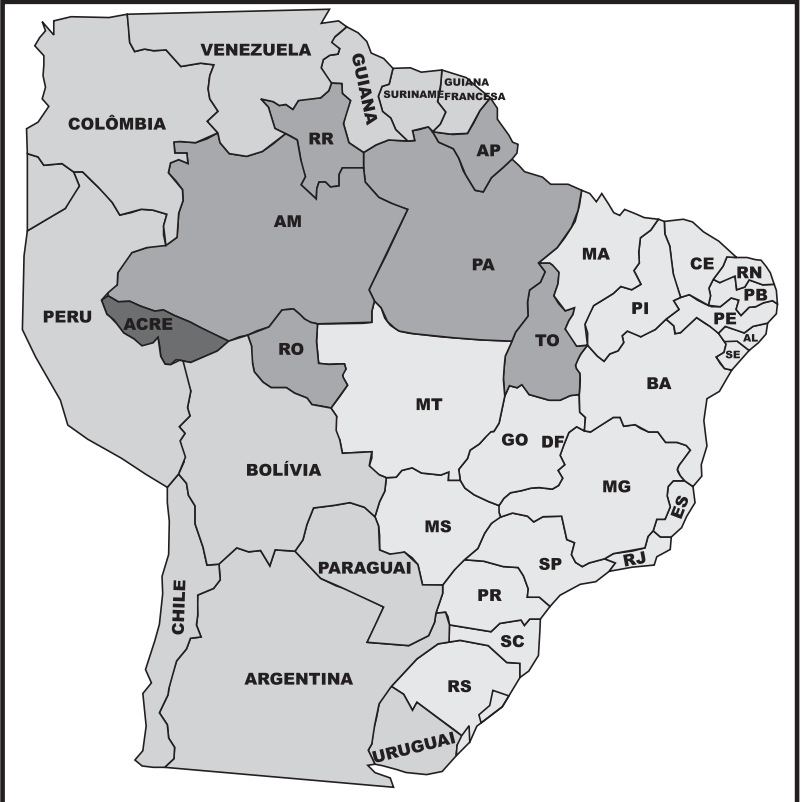

Figura 1 - Localização do Acre na região Amazônica, no Brasil e na América Latina.

fronteira com o sudoeste do Estado do Amazonas. Sua população em 2001 era de 30.000 habitantes, 55\% na área urbana ${ }^{14}$.

O bairro Cafezal na Cidade de Sena Madureira foi escolhido como uma amostra de conveniência da população urbana por ser referido como de ocupação antiga, grande em relação aos demais bairros da cidade $\left(10.500 \mathrm{~m}^{2}, 336\right.$ domicílios $)$ e supostamente heterogêneo em sua composição, aparentemente bem representativo da cidade. A área rural foi representada por sete comunidades ribeirinhas dos quatro principais rios da microrregião: Purus, Iaco, Caeté e Macauã (Figura 2). 0 bairro foi recenseado, visando o inquérito, com a cooperação da equipe da Fundação Nacional de Saúde (FUNASA) de Sena Madureira.

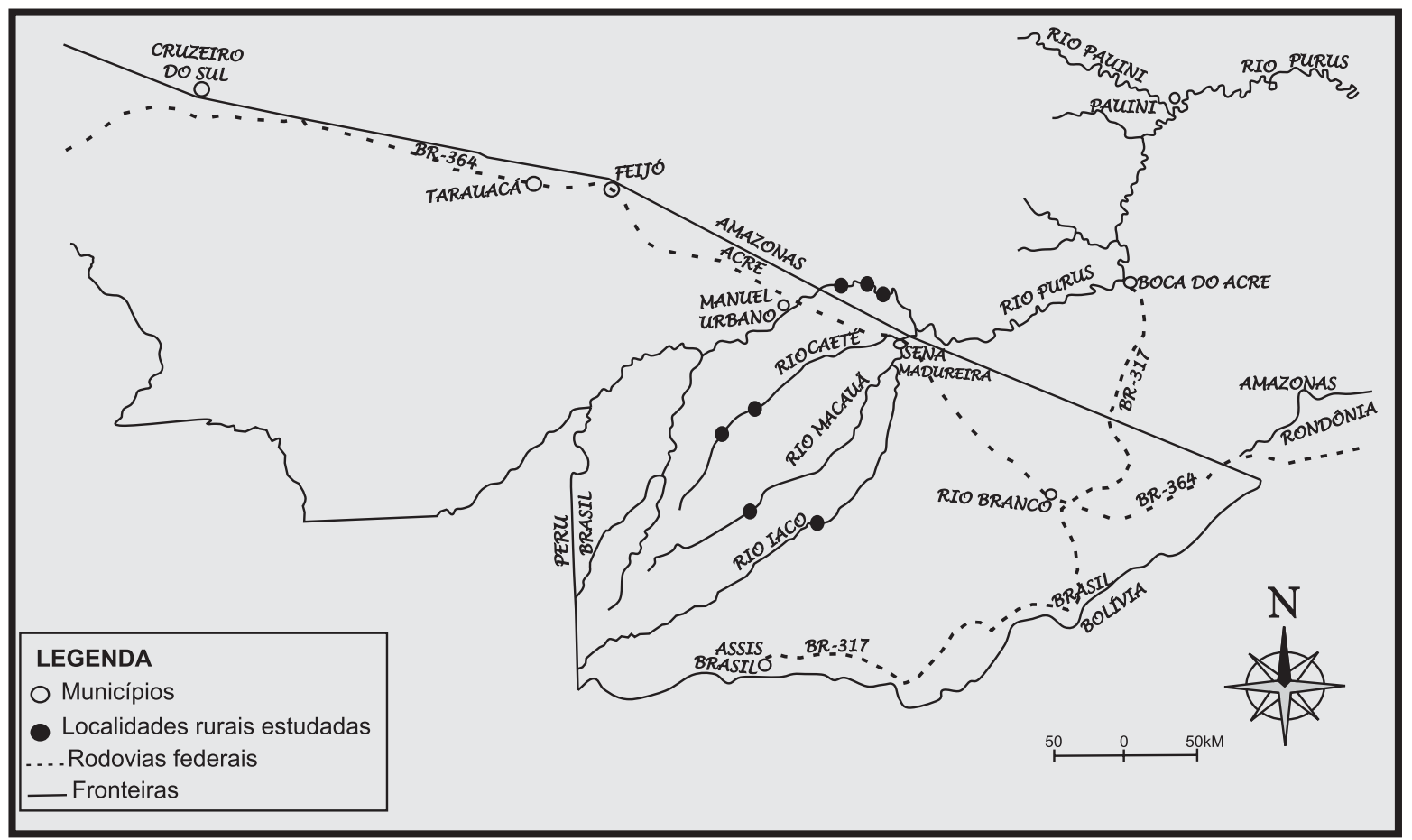

Figura 2 - Localização das comunidades ribeirinhas da amostra rural. Sena Madureira, Acre e sudoeste do Estado do Amazonas, Brasil, 2001. 
Foram realizados os cálculos de tamanho da amostra seguindo os princípios estabelecidos para variáveis dicotômicas e estudos descritivos através do aplicativo EPITABLE do programa Epi Info 6.04 (CDC, Atlanta \& OMS, Genebra). Considerou-se a estimativa de prevalência do inquérito nacional ${ }^{3}$, um nível de significância de 5\% e um efeito de desenho de 2. Para a área urbana admitiu-se um erro máximo (semi-amplitude do intervalo de confiança) de 2,5\% e para a área rural, de 3\%. A amostra mínima foi estimada em 444 indivíduos para a área urbana e 199 para a área rural. Foram incluídos no estudo apenas indivíduos acima de doze anos de idade.

As amostras de sangue foram coletadas por punção venosa entre janeiro e março de 2001. Os soros foram acondicionados em tubos Eppendorf, congelados a $-20^{\circ} \mathrm{C}$ e enviados ao Instituto de Patologia Tropical e Saúde Pública (IPTSP) da Universidade Federal de Goiás (UFG), onde foi realizado o estudo sorológico das amostras.

Todas as amostras foram inicialmente submetidas a dois testes em paralelo: ensaio imunoenzimático (ELISA), com intervalo de corte as absorbâncias de 0,9 a 1,1 e imunofluorescência indireta (IFI), com intervalo de corte as diluições de $1 / 20$ a $1 / 40$. Os soros que apresentaram resultados não reagentes pelas duas técnicas ou um resultado não reagente e um limítrofe foram considerados não infectados. Os que apresentaram: a) resultados discordantes; b) dois resultados limítrofes; ou c) um reagente e um limítrofe, foram considerados inconclusivos e re-testados. Foram re-testados também os inicialmente soropositivos (dois resultados reagentes). Nessa segunda etapa de testes repetiu-se a IFI nas mesmas condições anteriores, realizou-se o ELISA utilizando placas Nunc ${ }^{\circledR}$ (Copenhagen, Dinamarca) e a hemaglutinação indireta (HAI) com e sem a utilização de 2-mercapto-etanol (2ME) para eliminação de anticorpos naturais aglutinantes. 0 intervalo de corte para a HAI foram as diluições de 1/10 a 1/20 antes da incubação e a diluição de 1/8 foi o ponto de corte após incubação com 2ME.

Os antígenos utilizados para as reações sorológicas pelas técnicas de ELISA e IFI foram obtidos a partir de cultura da cepa Y de Trypanosoma cruzi em meio LIT. Os conjugados de anti-imunoglobulina humana $\mathrm{G}$ utilizados para a técnica de IFI, foram da Biolab ${ }^{\circledR}$, e para ELISA da Sigma ${ }^{\circledR}$. A técnica de HAI foi realizada com kits comerciais da Imunoserum ${ }^{\circledR}$. As reações de IFI foram examinadas através de epi-iluminação com microscópio de fluorescência Olympus ${ }^{\circledR}$ e as reações de ELISA lidas em espectrofotômetro Tecan Espectra ${ }^{\circledR}$.

Foram considerados casos (infecção chagásica) aqueles indivíduos que apresentaram, ao final da segunda etapa, reatividade no exame de seus soros para ao menos duas das técnicas utilizadas.

Foram coletadas através de entrevista individual as seguintes informações clínico-epidemiológicas: sexo, idade, ocupação, naturalidade, residência, história de viagens, queixas de palpitação, dispnéia, precordialgia, disfagia, odinofagia, pirose e obstipação.

Realizou-se ainda a busca de triatomíneos em dois peridomicílios (galinheiros) e um ecótopo silvestre (palmeira uricuri, Attalea sp) de cada comunidade ribeirinha incluída na amostra rural. As palmeiras escolhidas, por conveniência, distavam até 50 metros do peridomicílio estudado e foram examinadas por derrubada e dissecação da copa, com o auxílio de dois técnicos da FUNASA. Os triatomíneos capturados foram examinados no Serviço de Entomologia do Centro de Pesquisa Gonçalo Moniz (FIOCRUZ), na Bahia.

Foi construído um banco de dados no aplicativo estatístico SPSS $^{\circledR}$ versão 9.0 para Windows ${ }^{\circledR}$, onde foram realizadas as tabulações e análises estatísticas. Variáveis contínuas foram descritas através de média, mediana, desvio padrão e intervalo. Variáveis categóricas foram descritas através de proporções. A precisão das estimativas de prevalência foi descrita através de seus intervalos de confiança. A diferença entre os coeficientes de prevalência das áreas urbana e rural foi avaliada através da sobreposição de seus intervalos de confiança e pelo teste exato de Fisher, devido à ocorrência de frequiências esperadas menores que cinco.

0 estudo obedeceu aos princípios éticos contidos nas diretrizes da resolução 196/96 do Conselho Nacional de Saúde sobre pesquisa envolvendo seres humanos. 0 estudo foi considerado de risco mínimo, tendo sido identificados como potencialmente importantes os riscos advindos das complicações das punções venosas e aqueles relacionados com sigilo e confidencialidade das informações prestadas. Todos os participantes da pesquisa foram previamente informados sobre os objetivos, riscos e benefícios, individuais e coletivos, advindos de sua participação no estudo, tendo os mesmos consentido em participar e assinado o Termo de Consentimento Livre e Esclarecido. Os indivíduos identificados como infectados foram referenciados para acompanhamento no ambulatório de doenças infecciosas do Hospital Geral de Clínicas de Rio Branco.

\section{RESULTADOS}

Foram coletadas para o inquérito 1.055 amostras sangüíneas com as respectivas informações clínico-epidemiológicas, 844 constituindo a amostra urbana e 211 a amostra rural. A amostra populacional urbana teve média e mediana de idade de 35 e 29 anos, respectivamente (desvio padrão $=19$ anos e intervalo entre 12 e 91 anos) e a amostra populacional rural, média e mediana de 32 e 28 anos, respectivamente (desvio padrão = 16 anos e intervalo entre 12 e 80 anos).

Entre os indivíduos da amostra populacional urbana houve um predomínio do sexo feminino em todas as faixas de idade, enquanto na área rural, predominou o sexo masculino, especialmente na faixa de 30-39 e acima de 60 anos. A distribuição percentual do total de indivíduos, de acordo com as faixas etárias, é semelhante nas duas amostras, exceto na faixa acima de 60 anos, havendo maior participação de indivíduos idosos na amostra populacional urbana (Tabela 1).

Quanto à distribuição dos indivíduos incluídos na amostra rural, segundo seu local de moradia, observa-se que não é uniforme entre os diferentes rios. 0 rio Caeté representou 40,3\% da amostra rural com 85 indivíduos, seguido pelo rio Purus com 
Tabela 1 - Distribuição dos 1.055 indivíduos das amostras populacionais urbana e rural, segundo a faixa etária e o sexo, Sena Madureira, Acre e sudoeste do Estado do Amazonas, 2001.

\begin{tabular}{|c|c|c|c|c|c|c|c|c|c|c|c|c|}
\hline \multirow{3}{*}{$\begin{array}{l}\text { Faixa etária } \\
\text { (anos) }\end{array}$} & \multicolumn{6}{|c|}{ Urbana } & \multicolumn{6}{|c|}{ Rural } \\
\hline & \multicolumn{2}{|c|}{ masculino } & \multicolumn{2}{|c|}{ feminino } & \multicolumn{2}{|c|}{ total } & \multicolumn{2}{|c|}{ masculino } & \multicolumn{2}{|c|}{ feminino } & \multicolumn{2}{|c|}{ total } \\
\hline & $\mathrm{n}^{\mathrm{0}}$ & $\%$ & $\mathrm{n}^{0}$ & $\%$ & $\mathrm{n}^{\underline{0}}$ & $\%$ & $\mathrm{n}^{\underline{0}}$ & $\%$ & $\mathrm{n}^{\underline{0}}$ & $\%$ & $\mathrm{n}^{\underline{0}}$ & $\%$ \\
\hline $12-19$ & 91 & 10,8 & 140 & 16,6 & 231 & 27,4 & 31 & 14,7 & 26 & 12,3 & 57 & 27,0 \\
\hline 20-29 & 79 & 9,4 & 114 & 13,5 & 193 & 22,9 & 28 & 13,3 & 28 & 13,3 & 56 & 26,6 \\
\hline $30-39$ & 52 & 6,2 & 80 & 9,5 & 132 & 15,7 & 21 & 10,0 & 11 & 5,2 & 32 & 15,2 \\
\hline $40-49$ & 36 & 4,3 & 67 & 7,9 & 103 & 12,2 & 16 & 7,6 & 13 & 6,2 & 29 & 13,8 \\
\hline $50-59$ & 28 & 3,3 & 36 & 4,3 & 64 & 7,6 & 10 & 4,7 & 10 & 4,7 & 20 & 9,4 \\
\hline$\geq 60$ & 55 & 6,5 & 65 & 7,7 & 120 & 14,2 & 11 & 5,2 & 4 & 1,9 & 15 & 7,1 \\
\hline Ignorado & 1 & 0,1 & - & - & 1 & 0,1 & 2 & 0,9 & - & - & 2 & 0,9 \\
\hline Total & 342 & 40,5 & 502 & 59,5 & 844 & 100,0 & 119 & 56,4 & 92 & 43,6 & 211 & 100,0 \\
\hline
\end{tabular}

67 (31,8\%) indivíduos, rio Macauã com 44 (20,8\%) indivíduos e rio Iaco com 15 indivíduos representado $7,1 \%$ da amostra populacional rural (Tabela 2).

Tabela 2 - Distribuição dos 211 indivíduos da amostra populacional rural, segundo o local de moradia (localidade, rio e unidade da federação), Sena Madureira, Acre e sudoeste do Estado do Amazonas, 2001.

\begin{tabular}{lcc}
\hline Localidade & Número & Porcentagem \\
\hline Rio Purus (AM) & 28 & 13,3 \\
Descanso & 27 & 12,8 \\
São José & 12 & 5,7 \\
São Miguel & & \\
Rio Caeté (AC) & 68 & 32,2 \\
Cazumbá & 17 & 8,1 \\
Iracema & & \\
Rio Macauã (AC) & 44 & 20,8 \\
Liberdade & & 7,1 \\
Rio Iaco (AC) & 15 & 100,0 \\
São José & 211 & \\
\hline Total &
\end{tabular}

UF = Unidade da Federação; $\mathrm{AM}=$ Amazonas; $\mathrm{AC}=$ Acre
A habitação típica encontrada na área rural da região estudada possui piso $(96,7 \%)$ e paredes $(95,7 \%)$ de madeira, com cobertura de palha $(85,3 \%)$ ou, menos frequientemente, de zinco (12,3\%), não sendo encontrado domicílios que utilizassem barro batido em sua construção.

Foram capturados 21 exemplares de triatomíneos na copa das palmeiras, todos da espécie Rhodnius pictipes. Nenhum triatomíneo foi encontrado nos peridomicílios examinados. Só foi possível o estudo do conteúdo intestinal de um exemplar que se revelou negativo para a presença de flagelados (dados não apresentados em tabela).

Das 1.055 amostras de soros avaliadas, $134(12,7 \%)$ apresentaram resultados inicialmente inconclusivos e 13 (1,2\%) apresentaram-se inicialmente soropositivas (reagentes pelas duas técnicas). Nenhuma das amostras inicialmente inconclusivas se confirmou como de infectado. Dentre as amostras inicialmente soropositivas, apenas 9 se confirmaram como de indivíduos infectados. Dezesseis (1,5\%) amostras permaneceram com resultado duvidoso ao final do estudo (Figura 3).

Foram diagnosticados cinco casos de infecção chagásica na área urbana e quatro na área rural, gerando uma estimativa

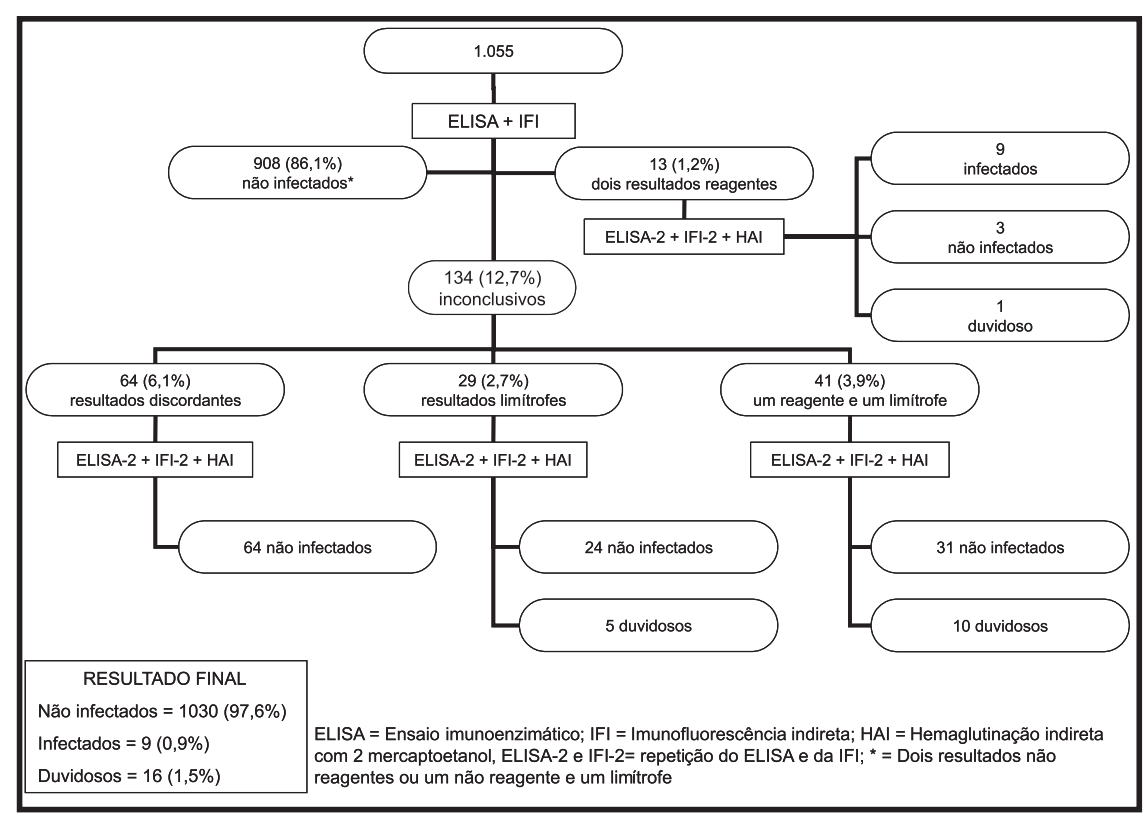

Figura 3 - Resultados dos exams sorológicos nas 1.055 amostras examinadas, Sena Madureira, Acre e Sudoeste do Estado do Amazonas, 2001. 
de prevalência de infecção pelo Trypanosoma cruzi de 0,6\% (IC 95\% 0,2- 1,4) para a área urbana e de 1,9\% (IC95\% 0,6-4,5) para a área rural (Tabela 3).

Os nove casos identificados (Tabela 4) possuem média e mediana de idade de $47,4 \mathrm{e} 46$ anos, respectivamente, com desvio padrão de 21 anos e intervalo entre 16 e 72 anos. Três são do sexo feminino. Todos são naturais da Região Amazônica. Dos cinco casos da área urbana, quatro são naturais de localidades ribeirinhas do rio Purus. Todos os quatro casos da área rural nasceram no rio Purus, um na cidade de Manoel Urbano e três em localidades ribeirinhas onde até hoje residem. A comunidade de São Miguel no rio Purus é o local de nascimento ou residência de quatro dos nove casos. Nesta localidade, ainda, dois casos foram identificados em um mesmo domicílio (dados não apresentado em tabela).

Somente um indivíduo referiu história de viagem para fora da Região Amazônica, em Goiânia-GO, em janeiro de 2001,
Tabela 3 - Resultados sorológicos reagentes e prevalência estimada de infecção pelo T. cruzi, nas áreas urbana e rural de Sena Madureira, Acre e sudoeste do Estado do Amazonas, 2001.

\begin{tabular}{lcccccc}
\hline & \multicolumn{2}{c}{ Soros } & Prevalência & IC 95\% para a & Valor de \\
\cline { 2 - 3 } Área & reagentes & examinados & $(\%)$ & proporção & p \\
\hline Urbana & 5 & 844 & 0,6 & $0,2-1,4$ & 0,85 \\
Rural & 4 & 211 & 1,9 & $0,6-4,5$ & \\
\hline
\end{tabular}

IC $95 \%$ = Intervalo de confiança de $95 \%$

uma única vez, para tratamento de cardiopatia de etiologia desconhecida, onde permaneceu por 19 dias. Esta paciente referiu palpitação e precordialgia em repouso, dispnéia aos médios esforços, disfagia para líquidos, e obstipação de até três dias. Referiu ainda, espontaneamente, história de febre de um mês de duração, há aproximadamente 28-30 anos. Este caso foi o único sorologicamente reagente a relatar história de disfagia, odinofagia e precordialgia.

\begin{tabular}{|c|c|c|c|c|c|c|c|c|c|}
\hline Informações & \multicolumn{5}{|c|}{ Urbana } & \multicolumn{4}{|c|}{ Rural } \\
\hline Sexo & fem & fem & masc & masc & masc & fem & masc & masc & masc \\
\hline Idade (anos) & 38 & 56 & 72 & 69 & 16 & 28 & 30 & 72 & 46 \\
\hline Naturalidade & Manaus & Purus & Purus & Purus & Purus & Purus & Purus & Purus & $\mathrm{MU}$ \\
\hline Localidade & NSA & São Brás & NM & NM & São Miguel & Descanso & São Miguel & São Miguel & NSA \\
\hline Residência & SM & SM & SM & SM & SM & Purus & Purus & Purus & Purus \\
\hline Localidade & NSA & NSA & NSA & NSA & NSA & Descanso & São Miguel & São Miguel & São Miguel \\
\hline Precordialgia & não & $\operatorname{sim}$ & não & não & não & não & não & não & não \\
\hline Disfagia & não & $\operatorname{sim}$ & não & não & não & não & não & não & não \\
\hline Dor esofágica & não & $\operatorname{sim}$ & não & não & não & não & não & não & não \\
\hline Pirose & não & não & não & não & não & não & sim & $\operatorname{sim}$ & não \\
\hline Obstipação*** & não & $\operatorname{sim}$ & $\operatorname{sim}$ & não & não & não & não & não & não \\
\hline
\end{tabular}

*para fora da região Amazônica; ** Obstipação $\geq 3$ dias. NM: Não mencionado; NSA: Não se aplica; SM: Sena Madureira; MU: Manuel Urbano

\section{DISCUSSÃo}

Este trabalho demonstrou que a prevalência de infecção chagásica na população adulta da área estudada, estimada através de sorologia, foi baixa.

Embora com tamanhos amostrais satisfatórios, nenhuma das duas áreas foi estudada através de amostras aleatórias simples, ideais para estimar parâmetros populacionais. Mesmo considerando-se um efeito de desenho que poderia corrigir pelo menos parcialmente as distorções, chama a atenção em especial a distribuição desigual da amostra rural entre os diferentes rios da região, resultante da amostragem de conveniência. Pode-se no caso da amostra urbana, com algum grau de segurança, supor que os diversos extratos populacionais da área urbana de Sena Madureira possam se distribuir aleatoriamente pela população do bairro do Cafezal, dado seu tamanho e sua suposta heterogeneidade, aproximando-se assim de uma amostra aleatória simples. A distribuição das duas amostras populacionais segundo o sexo, mostrando o predomínio feminino na área urbana e 0 masculino na rural, é indício de representatividade uma vez que é esta a distribuição observada nas populações urbana e rural do município de Sena Madureira ${ }^{14}$.

As estimativas de prevalência encontradas para as áreas urbana e rural apresentaram intervalos de confiança superponíveis, e teste de hipótese negativo para diferença entre as proporções, o que não permite identificá-las como significativamente diferentes.

Os nove indivíduos identificados como infectados são de ambos os sexos e com idades variando de 16 a 72 anos. Sete são naturais de comunidades rurais do rio Purus e um é natural da cidade de Manuel Urbano também na bacia do alto Purus. A comunidade de São Miguel é a residência de três dos quatro casos da área rural e o local de nascimento de pelo menos um 
dos casos da área urbana. 0 quarto caso rural é procedente da comunidade de Descanso, também no rio Purus.

Tais achados, embora não possam com segurança deixar de ser atribuídos ao acaso, dado à pequena quantidade de casos, não deixam de chamar a atenção, assim como chama a atenção também pela curiosidade, embora cientificamente irrelevante, $o$ fato de ter sido frequiente o relato entre as comunidades ribeirinhas de que o Purus é de todos os rios da região o mais doente.

A aparente aglomeração dos casos reagentes, mais freqüentes em um dentre os quatro rios estudados, em uma das três localidades deste mesmo rio, e em uma casa dentre várias de uma mesma localidade também é digna de atenção. Tem sido notada a ocorrência na região Amazônica, ora de um padrão de transmissão característico de micro epidemias familiares, fortemente sugestivo de transmissão por fonte comum, como a via oral $^{23}$, ora de um padrão de transmissão de características claramente ocupacionais ${ }^{5}$, ambas com tendência a ocorrer de forma aglomerada. Os achados deste estudo estão portanto, em princípio, em consonância com tais padrões, sendo necessário testar-se esta hipótese em um estudo posterior.

Dos nove casos identificados, oito nunca saíram da região Amazônica. 0 único caso que referiu ter saído, tem história de viagem única, recente (janeiro de 2001) e breve (19 dias) a GoiâniaG0 para tratamento de cardiopatia, sendo possível, portanto, concluir-se, em princípio, pela autoctonia de sua infecção. Era uma paciente feminina, de 56 anos que se queixou de disfagia, odinofagia e precordialgia, sendo o único caso com doença possivelmente atribuível à infecção crônica pelo Trypanosoma cruzi. Esta paciente referiu ainda espontaneamente história de febre de um mês de duração há aproximadamente 28-30 anos, podendo esta sintomatologia ter representado a fase aguda de sua infecção.

O estudo entomológico, longe de ser um verdadeiro inquérito, pôde identificar Rhodnius pictipes nos ecótopos silvestres próximos, mas não triatomíneos peridomiciliados. Nada se pôde concluir sobre a infecção natural dos triatomíneos pelo exame de apenas um exemplar.

Os resultados indicam a necessidade de acompanhamento de todos os indivíduos identificados como infectados, sendo imperativa a realização de exames parasitológicos, eletrocardiográficos e radiológicos, bem como a avaliação posterior do possível benefício individual de cada um em se submeter ao tratamento específico.

\section{AGRADECIMENTOS}

Os autores manifestam seu reconhecimento e agradecimento às Dras. Suelene Brito do Nascimento Tavares e Rosângela Amaral de Oliveira, pelo cuidado e dedicação ao laborioso trabalho de bancada com as técnicas sorológicas, indispensável à realização desse estudo.

\section{REFERÊNCIAS}

1. Barata JMS, Rocha RM, Rodrigues VLCC, Ferraz-Filho AN. Primeiro caso autóctone de tripanossomíase americana do Estado do Acre (Brasil) e sua correlação com as cepas isoladas do caso humano e de triatomíneos silvestres da área. Revista de Saúde Pública 22:401-410, 1988.
2. Barreto MP. Reservatórios do Trypanosoma cruzi nas Américas. Revista Brasileira de Malariologia e Doenças Tropicais 16:527-552, 1964.

3. Camargo ME, Silva GR, Castilho EA, Silveira AC. Inquérito sorológico da prevalência de infecção chagásica no Brasil, 1875/1980. Revista do Instituto de Medicina Tropical de São Paulo 26:192-204, 1984.

4. Coura JR. Doença de Chagas como endemia na Amazônia Brasileira: risco ou hipótese. Revista da Sociedade Brasileira de Medicina Tropical 23:67-70, 1990.

5. Coura JR, Barret TV, Naranjo MA. Ataque de populações humanas por triatomíneos silvestres no Amazonas: uma nova forma de transmissão de infecção chagásica? Revista da Sociedade Brasileira de Medicina Tropical 27:251-253, 1994.

6. Coura JR, Celano VS, Santos LC, Gonçalves AQ, Spata MCD, Moreira CJC, Bóia MN, Fernandes 0, Junqueira ACV. Transmissão vetorial da infecção chagásica na Amazônia Brasileira. Revista da Sociedade Brasileira de Medicina Tropical 33 (supl II):29-31, 2000.

7. Coura JR, Junqueira ACV, Bóia MN, Fernandes O. Chagas' disease: from bush to huts and houses. Is it the case of the Brazilian Amazon? Memórias do Instituto Oswaldo Cruz 94 (supl I):379-384, 1999.

8. Coura JR, Junqueira ACV, Giordano CM, Funatsu RK. Chagas'disease in the Brazilian Amazon I- a short review. Revista do Instituto de Medicina Tropical de São Paulo 36:363-368, 1994.

9. Deane LM. Animal reservoirs of Trypanosoma cruzi in Brazil. Revista Brasileira de Malariologia e Doenças Tropicais 16:27-48, 1964.

10. Deane LM. Tripanosomídeos de mamíferos da região Amazônica IV-Hemoscopia e xenodiagnóstico de animais silvestres da estrada Belém-Brasília. Revista do Instituto de Medicina Tropical de São Paulo 9:143-148, 1967.

11. Dias JCP. Epidemiologia. In: Brener Z, Andrade ZA, Barral-Netto M (eds) Trypanosoma cruzi e Doença de Chagas, $2^{\text {a }}$ edition, Guanabara Koogan, Rio de Janeiro, p. 48-74, 2000.

12. Dias JCP, Prata A, Schofield CJ. Doença de Chagas na Amazônia: esboço da situação atual e perspectivas de prevenção. Revista da Sociedade Brasileira de Medicina Tropical 35:669-678, 2002.

13. Feitosa VR. Implantação de um sistema de Vigilância Epidemiológica (VE) de doença de Chagas na Amazônia. Revista da Sociedade Brasileira de Medicina Tropical 28 (supl III):84-87, 1995.

14. Fundação Instituto Brasileiro de Geografia e Estatística. Censo demográfico. sinopse preliminar. http://www.ibge.org.br (acessado em 16/Jul/2001), 2000.

15. Governo do Estado do Acre. Programa Estadual de Zoneamento EcológicoEconômico do Estado do Acre. Zoneamento Ecológico-Econômico - documento final. SECTMA, Rio Branco, 2000.

16. Luitgards-Moura JF, Vargas AB, Almeida CE, Magno-Esperança G, Agapito-Souza R, Folly-Ramos E, Costa J, Tsouris P, Rosa-Freitas MG. A Triatoma maculata (Hemiptera, Reduviidae, Triatominae) population from Roraima, Amazon region, Brazil, has some bionomic characteristics of a potential Chagas disease vector. Revista do Instituto de Medicina Tropical de São Paulo 47:131-137, 2005.

17. Shaw J, Lainson R, Fraiha H. Considerações sobre a epidemiologia dos primeiros casos autóctones de doença de Chagas registrados em Belém, Pará, Brasil. Revista de Saúde Pública de São Paulo 3:153-157, 1969.

18. Sherlock I. Epidemiology and dinamics of the vectorial transmission of Chagas disease. Memórias do Instituto Oswaldo Cruz 94 (supl I):385-386, 1999.

19. Silveira AC. Situação do controle da transmissão vetorial da doença de Chagas no Brasil. Revista da Sociedade Brasileira de Medicina Tropical 30 (supl I):42-44, 1997.

20. Silveira AC, Vinhaes M. Doença de Chagas: aspectos epidemiológicos e de controle. Revista da Sociedade Brasileira de Medicina Tropical 31 (supl II): 15-60, 1998

21. Valente VC. Potencial for domestication of Panstrongylus geniculatus (Latreille, 1811) (Liemiptera, Reduvidae, Triatominae) in the munnicipality of Muaná, Marajó Island, State of Pará, Brazil. Memórias do Instituto Oswaldo Cruz 94 (supl I):399-400, 1999.

22. Valente SAS, Valente VC, Fraiha-Neto H. Considerações sobre a epidemiologia e transmissão da doença de Chagas na Amazônia Brasileira. Revista da Sociedade Brasileira de Medicina Tropical 32 (supl II):51-55, 1999. 
23. Valente SAS, Valente VC, Pinto AYN, Fraiha-Neto H. Microepidemia familiar e transmissão oral da doença de Chagas na Amazônia Brasileira. Memórias do Instituto Oswaldo Cruz 95 (supl II):69-72, 2000

24. Viana S, Farias E, Lima F, Batista L, Vieira A, Silva L, Lobato C, Nascimento S, Chalub S. Doença de Chagas no Estado do Acre: registro de três casos de miocardiopatia chagásica aguda autóctone no Município de Rio Branco. Revista da Sociedade Brasileira de Medicina Tropical 27(supl I):77, 1994

25. World Health Organization. Control of Chagas disease. Report of a WHO expert committee. WHO Technical Report Series 811. Geneva, 1991. 